\title{
Experimental opportunities of ChPT at J-PARC
}

\author{
Itaru Nakagawa*t \\ RIKEN/RIKEN-BNL Research Center \\ E-mail: itaruebnl.gov
}

Japan Proton Accelerator Research Complex (J-PARC) is the new high luminosity accelerator complex facility being constructed about $130 \mathrm{~km}$ north-east of Tokyo. About $2.5 \times 10^{14}$ protons/sec will be accelerated up to $50 \mathrm{GeV}$ at when the power of $0.75 \mathrm{MW}$ is achieved. At the 50-GeV Proton Synchrotron (PS), nuclear/particle physics experiments using kaon beams, antiproton beams, hyperon beams and primary proton beams are planned. The commission of the first experiments using secondary kaon beam $\left(E_{\mathrm{K}}<2 \mathrm{GeV}\right)$ will be started towards the end of 2009. This new hadron facility will provide new experimental opportunities for stringent test of ChPT. For example, the meson polarizability measurements can be one of suitable experiments at JPARC, taking advantage of secondary meson beam from the world's highest luminosity proton beam. The polarizabilities of pion and kaon characterize the deformation of the pion and kaon in an external electromagnetic field and can be predicted by ChPT in high precision. The feasibility of such a measurement using J-PARC facility is studied.

6th International Workshop on Chiral Dynamics

July 6-10 2009

Bern, Switzerland

\footnotetext{
*Speaker.

${ }^{\dagger}$ Mailing Address:Radiation Laboratory, 2-1 Hirosawa, Wako, Saitama 351-0198, Japan
} 


\section{INTRODUCTION}

The values of the electric and magnetic polarizabilities depend on the rigidity of the composite particle and provide important information of the internal structure. The meson sector of polarizabilities, are particular of interest because they are calculable in ChPT in high precision [1] (One-loop and two-loop[2],[3] corrections are predicted to be small) for pion electric $\alpha_{\pi}$ and magnetic $\beta_{\pi}$ polarizabilities, therefore polarizabilities are considered to be a vital testing ground for theories of low-energy QCD chiral dynamics.

While proton polarizabilities are reasonably well measured[4] through Compton scattering as represented by diagram (a) in Fig.1, the knowledge of the polarizabilities of pion and kaon are way behind that of proton. The determination of the polarizabilities is experimentally quite challenging due to the lack of free neither pion nor kaon targets. Thus the normal Compton diagram (a) can not be used for pion and kaon cases. Instead, one can use (b) meson pair production via photo fusion, (c) radiative meson photo production, (d) radiative meson scattering (Primakoff scattering). High energy $(10 \sim 40 \mathrm{GeV})$ pion and kaon scatter from a virtual photon in the Coulomb field of the target nucleus make accessible to the polarizability through the diagram (d). This approach has certain advantages due to the clear separation of the Primakoff scattering amplitude (very forward boosted peak) and strong interaction backgrounds. The only existing Primakoff datum is from Serpukhov experiment and their result[7] $\alpha_{\pi}-\beta_{\pi}=(15.6 \pm 7.8) \times 10^{-4} \mathrm{fm}^{3}$, disagree with two-loop order ChPT prediction[3] $(5.7 \pm 1.0) \times 10^{-4} \mathrm{fm}^{3}$ by two sigma. The latest pion polarizabilities were measured at Mainz through the interaction (c) after difficult extraction of the signal out of resonance backgrounds, and disagree with ChPT prediction by two sigma[8]. The polarizabilities extracted[5] from photo fusion data[6] $\alpha_{\pi}-\beta_{\pi}=\left(13.0_{-1.9}^{+2.6}\right) \times 10^{-4} \mathrm{fm}^{3}$ again disagree with ChPT prediction. In order to address the issue of this long standing discrepancies between experiments and ChPT, new high precision experiments with cleaner extraction of pion polarizabilities has been highly desired. New generation Primakoff experiment is prepared at COMPASS using $190 \mathrm{GeV}$ pion beam[9]. In this documentation, I would like to study another Primakoff experiment at J-PARC taking advantage of the secondary meson beam from world's highest intensity proton beam.

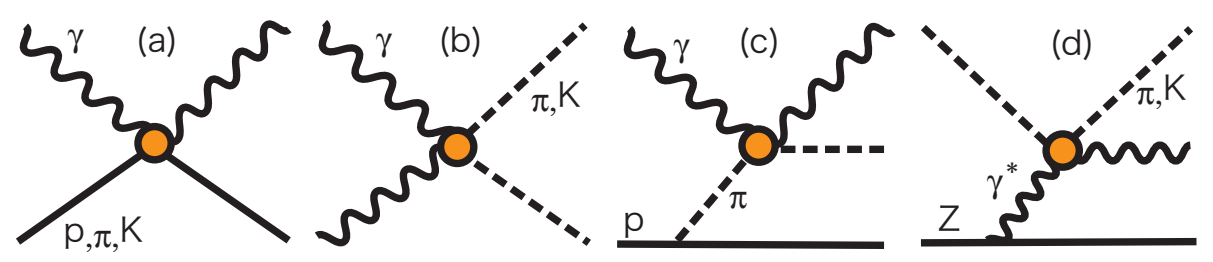

Figure 1: Compton diagram sensitive to the polarizability.

\section{J-PARC Facility}

The Japan Proton Accelerator Complex, J-PARC [10], is a high-intensity proton accelerator facility, under construction at Tokai, Japan. Fig. 2 shows bird's eye view of the J-PARC construction site. J-PARC has a cascade accelerator complex: the linear accelerator (Linac), the 3-GeV 
rapid cycling synchrotron and the $50 \mathrm{GeV}$ synchrotron. The experimental facilities are the Materials and Life Science Facility using muon and neutron beams produced by the $3 \mathrm{GeV}$ proton beams, the Hadron Hall, which utilizes slow-extracted proton beams from the $50 \mathrm{GeV}$ synchrotron, and the neutrino beamline. The goals of J-PARC are nuclear and particle physics with the $50 \mathrm{GeV}$ synchrotron, materials and life sciences with neutron and muon beams produced at the $3 \mathrm{GeV}$ synchrotron, and R\&D for nuclear transmutation using beams from the linear accelerator.

J-PARC aims to be the high-intensity frontier. J-PARC will have the proton beam power of 1 MW with the $3 \mathrm{GeV}$ synchrotron for MLF, and $0.75 \mathrm{MW}$ for the $50 \mathrm{GeV}$ facilities (Hadron Hall and neutrino beamline). As can be seen from Fig. 3, the beam power of J-PARC is almost one order higher than the existing accelerator facilities. The design performance of the $50 \mathrm{GeV}$ synchrotron is listed in Table 1.

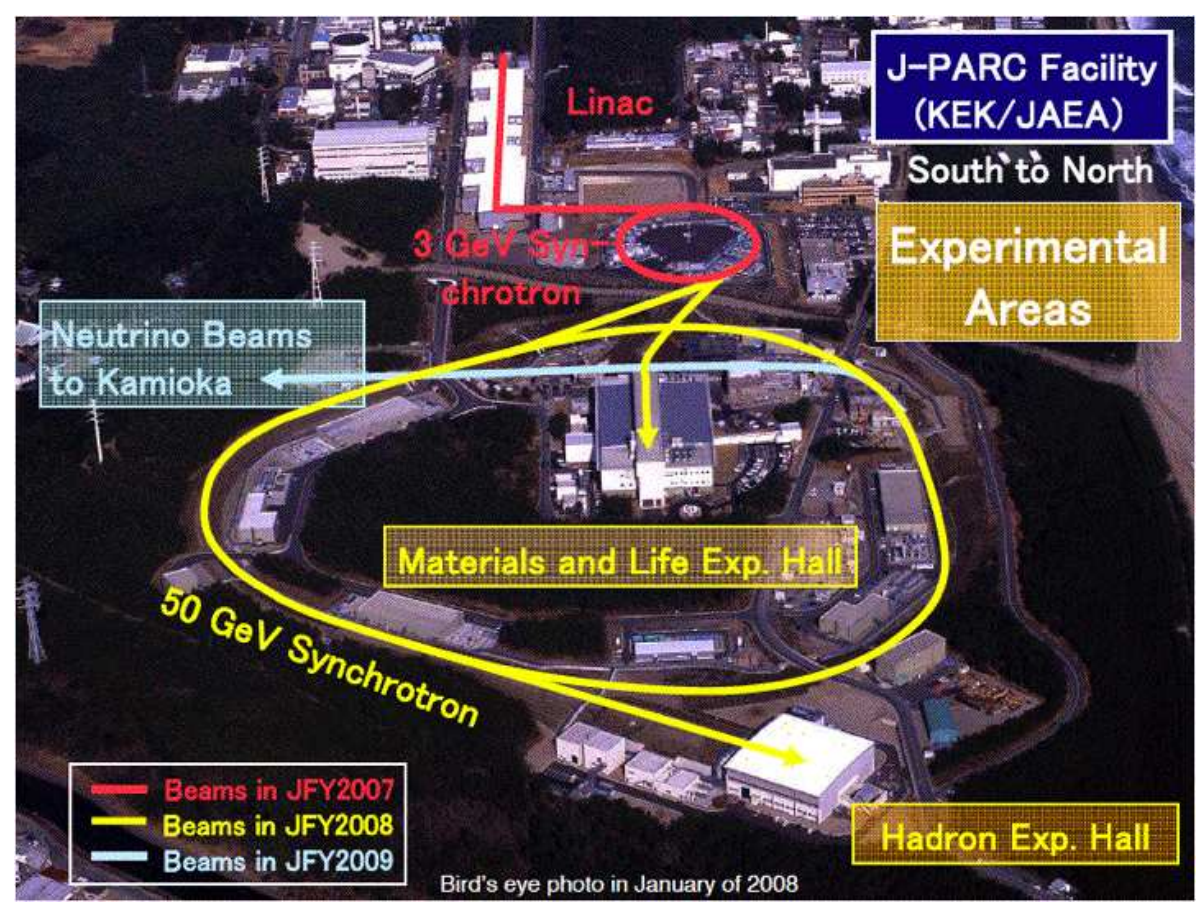

Figure 2: J-PARC construction site in bird's eye view as of 2008.

Table 1: Major performance parameters of J-PARC.

\begin{tabular}{cccccc}
\hline \hline & Beam Energy & Repetition Rate & Intensity & Current & Beam power \\
\hline Design Goal & $50 \mathrm{GeV}$ & $0.3 \mathrm{~Hz}$ & $3.3 \times 10^{14} \mathrm{ppp}$ & $15 \mu \mathrm{A}$ & $750 \mathrm{~kW}$ \\
\hline \hline
\end{tabular}

Nuclear physics experiments are to be mostly conducted in the hadron hall utilizes the slowextracted proton beams from the $50 \mathrm{GeV}$ synchrotron. At the beginning of the phase-1 (funded) operation, from the year of 2009 , only one secondary beam line, K1.8BR, will be ready. The day 1 experiments will be started towards the end of 2009 using the $1.8 \mathrm{GeV}$ secondary beam 


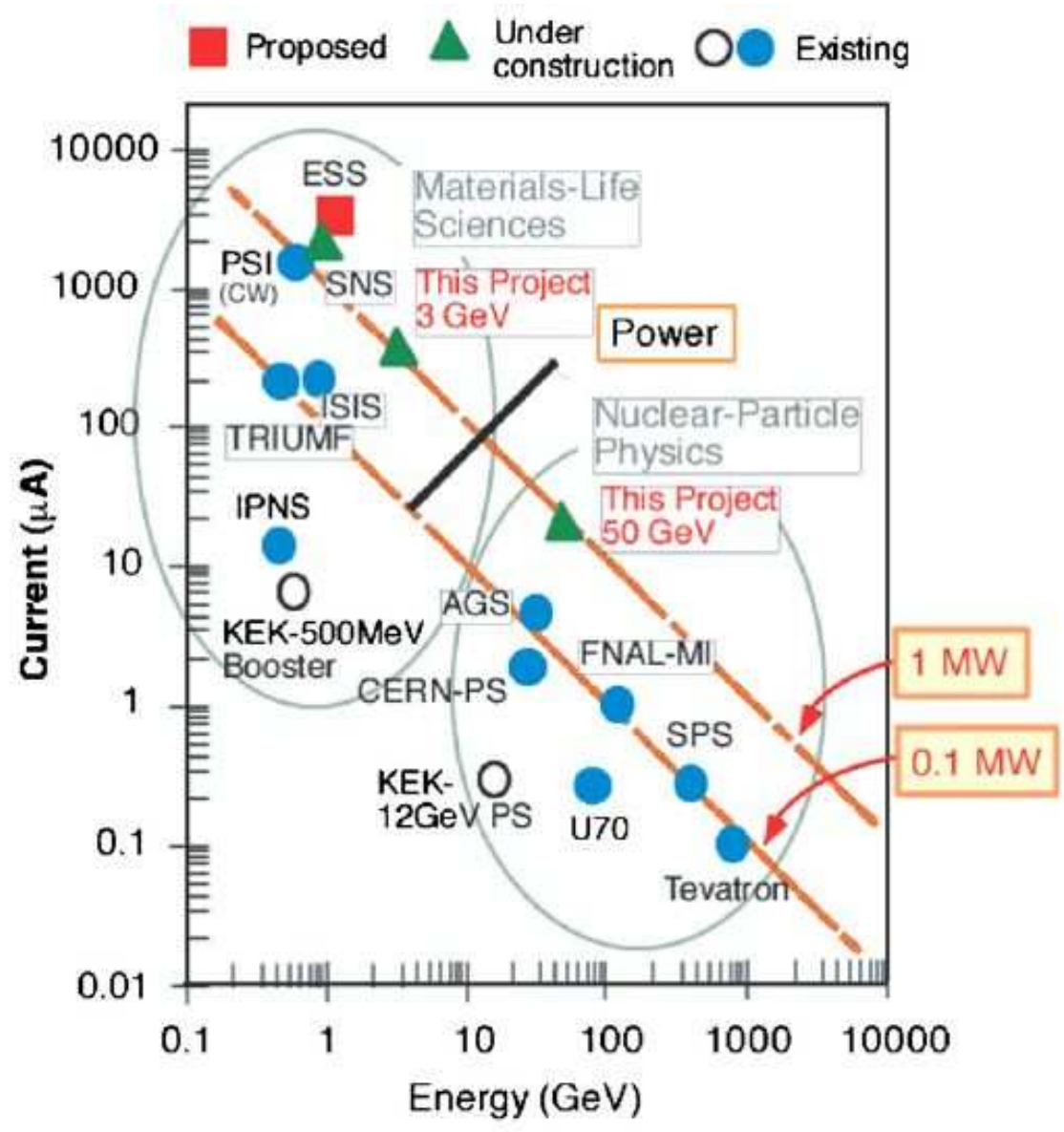

Figure 3: World proton accelerators and their beam power. The product of the beam current (vertical axis) and the energy (horizontal axis) is the beam power. J-PARC aims to be at the frontier of beam power.

line (K1.8BR) and other beam lines, such as $\mathrm{K} 1.8, \mathrm{~K} 1.1 / 0.8, \mathrm{KL}$, and the high-momentum beam line[11] will be constructed one after another depending on the budget situation in coming years. Because the size of the hadron hall is very much limited, extension of the hall downstream is planned from the beginning of the J-PARC project. The Primakoff experiment may require such an additional space.

\section{SECONDARY BEAM INTENSITY}

In this section, the secondary pion and kaon beam intensities were estimated using SanfordWang formula[12] for $50 \mathrm{GeV}^{1}$ and $10^{14}$ intensity proton beam. The formula predicts the angular $\theta$ and momentum $p$ distribution of secondaries in the laboratory system with phenomenological form:

\footnotetext{
${ }^{1}$ Sanford-Wang formula was designed to fit data between the proton beam energy of 10 and $35 \mathrm{GeV}$, therefore the prediction precision at $50 \mathrm{GeV}$ is limited by the exterpolation of applicable range[13].
} 


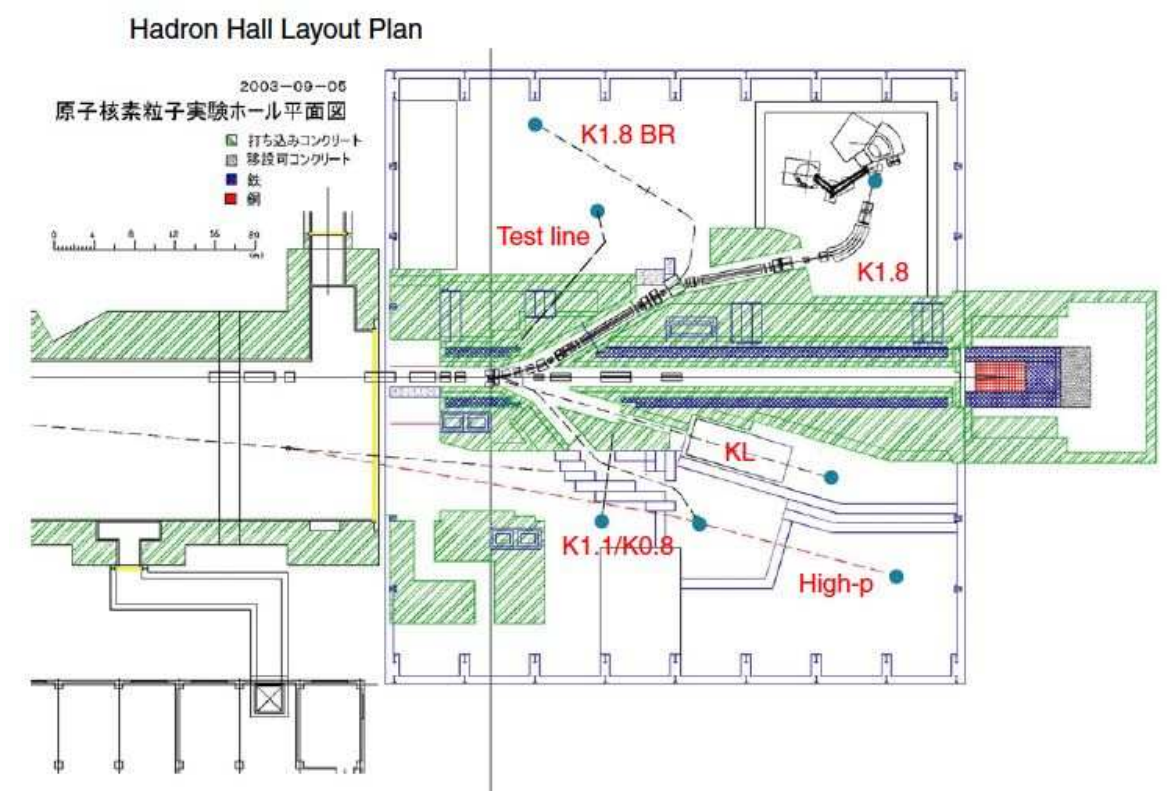

Figure 4: Layout plan for the beam lines and spectrometers in the hadron hall.

$$
\frac{d^{2} \sigma}{d p d \Omega}=A p^{B}\left(1-\frac{p}{p_{i}}\right) \times \exp \left[-\frac{C p^{D}}{p_{i}^{E}}-F \theta\left(p-G p_{i} \cos ^{H} \theta\right)\right]
$$

where $p_{i}$ is the momentum of the incident protons. $\mathrm{A} \sim \mathrm{H}$ are parameters unique to the species of secondary particles[14] (don't depend on the target nucleus) and are summarized in Table 2.

Table 2: Parameters of Sanford Wang's formula.

\begin{tabular}{ccccccccc}
\hline \hline secondary particle & $\mathrm{A}$ & $\mathrm{B}$ & $\mathrm{C}$ & $\mathrm{D}$ & $\mathrm{E}$ & $\mathrm{F}$ & $\mathrm{G}$ & $\mathrm{H}$ \\
\hline$\pi^{+}$ & 1.092 & 0.6458 & 4.046 & 1.625 & 1.656 & 5.029 & 0.1722 & 82.65 \\
$\pi^{-}$ & 0.821 & 0.5271 & 3.956 & 1.731 & 1.617 & 4.735 & 0.1984 & 88.75 \\
$K^{+}$ & 0.05597 & 0.6916 & 3.744 & 4.520 & 4.190 & 4.928 & 0.1922 & 50.28 \\
$K^{-}$ & 0.02210 & 1.323 & 9.671 & 1.712 & 1.643 & 4.673 & 0.1686 & 77.27 \\
\hline \hline
\end{tabular}

From given production cross section determined above, the yield $Y$ of secondary particles for proton beam intensity is calculated

$$
Y=N_{\mathrm{p}} N_{\mathrm{t}} \frac{d^{2} \sigma}{d p d \Omega} \Delta \Omega \Delta p
$$

where $N_{\mathrm{p}}$ and $N_{\mathrm{t}}$ are number of incident and target particles, respectively. Since Primakoff requires higher energy, yield estimate was done for two high secondary meson momentums, i.e. 30 and 40 $\mathrm{GeV}$. The previous Primakoff polarizability experiment at IHEP[7] was carried out at the secondary 
$\pi^{-}$momentum of $40 \mathrm{GeV}$. The acceptance $\Delta \Omega \Delta p / p$ was assumed $0.2 \mathrm{msr} \%$. Resulting yields at the production angles $4^{\circ}$ for $2 \%$ and $2^{\circ}$ for $10 \%$ loss copper target are summarized in Table 3 .

Table 3: Secondary meson production cross section and yield estimate with incident proton beam energy 50 $\mathrm{GeV}$ at the production angle $4^{\circ}$ and $2^{\circ}$ using $2 \%$ and $10 \%$ loss copper target, respectively.

\begin{tabular}{c|c|cc|cc}
\hline \hline \multicolumn{2}{c|}{} & \multicolumn{2}{c|}{$\theta=4^{\circ}, 2 \%$ loss } & \multicolumn{2}{c}{$\theta=2^{\circ}, 10 \%$ loss } \\
\hline secondary particle & $\begin{array}{c}p \\
{[\mathrm{GeV} / \mathrm{c}]}\end{array}$ & $\begin{array}{c}d \sigma / d p d \Omega \\
{[\mathrm{mb} / \mathrm{sr} / \mathrm{GeV} / \mathrm{c}]}\end{array}$ & $\begin{array}{c}\text { rate } \\
{[\mathrm{Hz}]}\end{array}$ & $\begin{array}{c}d \sigma / d p d \Omega \\
{[\mathrm{mb} / \mathrm{sr} / \mathrm{GeV} / \mathrm{c}]}\end{array}$ & $\begin{array}{c}\text { rate } \\
{[\mathrm{Hz}]}\end{array}$ \\
\hline$\pi^{+}$ & 30 & 0.20 & $3.1 \times 10^{4}$ & 14 & $1.0 \times 10^{7}$ \\
$\pi^{+}$ & 40 & 0.0014 & $2.9 \times 10^{2}$ & 0.57 & $6.0 \times 10^{5}$ \\
$\pi^{-}$ & 30 & 0.083 & $1.3 \times 10^{4}$ & 4.0 & $3.0 \times 10^{6}$ \\
$\pi^{-}$ & 40 & 0.00034 & $6.9 \times 10^{1}$ & 0.086 & $8.5 \times 10^{4}$ \\
$K^{+}$ & 30 & 0.030 & $4.4 \times 10^{3}$ & 1.4 & $1.0 \times 10^{6}$ \\
$K^{+}$ & 40 & 0.00002 & $3.1 \times 10^{0}$ & 0.0040 & $4.0 \times 10^{3}$ \\
$K^{-}$ & 30 & 0.0017 & $2.6 \times 10^{2}$ & 0.088 & $6.5 \times 10^{4}$ \\
\hline \hline
\end{tabular}

In general, the Primakoff experiment has advantage to run with higher momentum beam in terms of gain in cross section and clearer kinematical separation from backgrounds via strong interactions. However, the intensity of the secondary meson beam becomes strong constraint as presented in Table 3 due to drastic suppression of production yield by almost two order of magnitudes from $p=30 \mathrm{GeV}$ to $40 \mathrm{GeV}$. It is quite infeasible to design Primakoff experiment with secondary meson rate of in the order of $10^{2} \mathrm{~Hz}$. In order to obtain decent intensity of secondary beam in higher momenta, it is important to collect secondary particles at smaller production angle, such as $2^{\circ}$. A beam separation device for smaller angle with wide acceptance, such as a septum magnet, requires $R \& D$ studies[11]. $10 \%$ radiation loss target also requires additional radiation shielding. With this condition, pion rate in the order of $10^{5} \mathrm{~Hz}$ can be expected. Expected Primakoff yield is calculated for $\pi^{+}$rate of $0.6 \mathrm{MHz}$ at the secondary $\pi^{+}$energy of $40 \mathrm{GeV}$ in the next section.

\section{PRIMAKOFF YIELD ESTIMATE}

In this section, Primakoff yield is estimated from the secondary pion beam intensity estimated previously. First, the Compton-effect cross section in pion rest frame for structure-less pion $\left(\alpha_{\pi}=\right.$ $\beta_{\pi}=0$ ) is given[7]:

$$
\begin{aligned}
\frac{d^{2} \sigma}{d \omega_{1} d \cos \theta_{\gamma \gamma}} & =\frac{\alpha Z^{2}}{\pi \omega_{1}}\left[\ln \frac{t_{\max }}{t_{\min }}-1+\frac{t_{\min }}{t_{\max }}\right]\left[F_{\mathrm{A}}(t)\right]^{2} \frac{d \sigma_{\gamma}\left(\omega_{1}\right)}{d \cos \theta_{\gamma \gamma}} \\
\frac{d \sigma_{\gamma}\left(\omega_{1}\right)}{d \cos \theta_{\gamma \gamma}} & =\frac{\pi \alpha^{2}}{m_{\pi}^{2}} \frac{1+\cos ^{2} \theta_{\gamma \gamma}}{\left[1+\frac{\omega_{1}}{m_{\pi}}\left(1-\cos \theta_{\gamma \gamma}\right)\right]^{2}}
\end{aligned}
$$

where $Z, \omega_{1}$ and $\cos \theta_{\gamma \gamma}$ are target nucleus charge, incident (virtual) photon energy, and scattered photon angle, respectively. $t_{\min }$ and $t_{\max }$ are minimal and maximum values of the 4-momentum 
transfer squared, the first $t_{\min }$ is determined by the kinematics of the experiment $\left(t_{\min }=m_{\pi}^{2} \omega_{1}^{2} / E_{\pi}\right.$, $E_{\pi}$ : incident pion energy) and the second one is determined by the region where Coulomb scattering dominates strong interaction backgrounds. Since small $t$ region is considered here, the electromagnetic form factor of target nucleus was set $F_{\mathrm{A}}(t) \approx 1$. For pion incident energy $40 \mathrm{GeV}$, which is same energy for the existing Primakoff experiment at Serpukhov, kinematic constraints were estimated from that experiment[7] assuming similar acceptance coverage at J-PARC. For the average cross section, the central kinematic variables are chosen follows: $\cos \theta_{\gamma \gamma}=-1, \omega_{1}=0.3$ $\mathrm{GeV}, t_{\max }=6 \times 10^{-4}\left[(\mathrm{GeV} / \mathrm{c})^{2}\right]$. The resulting average Primakoff cross section for Carbon $(Z=6)$ target is

$$
\frac{d^{2} \sigma}{d \omega_{1} d \cos \theta_{\gamma \gamma}}=2.4 \times 10^{-31}\left[\mathrm{~cm}^{2} / \mathrm{GeV}\right]
$$

Expected Primakoff yield is calculated for incident pion intensity of $0.5 \mathrm{kHz}$ at $40 \mathrm{GeV}$ as follows:

$$
\begin{aligned}
Y_{\text {Primakoff }} & =\frac{d^{2} \sigma}{d \omega_{1} d \cos \theta_{\gamma \gamma}} N_{\mathrm{t}} N_{\pi, \mathrm{K}} \Delta \omega_{1}\left(\Delta \cos \theta_{\gamma \gamma}\right) \varepsilon \\
N_{\mathrm{t}} & : 0.25 X_{\text {rad }} \text { Carbon } \sim 5.3 \times 10^{23} \\
N_{\pi, \mathrm{K}} & =0.6[\mathrm{MHz}]: \text { secondary pion/kaon rate } \\
\Delta \omega_{1} & =0.5 \mathrm{GeV} \\
\Delta \cos \theta_{\gamma \gamma} & =0.8 \\
\varepsilon & : \text { Detector efficiencies and DAQ uptime }
\end{aligned}
$$

where $\Delta \omega_{1}=0.5 \mathrm{GeV}$ and $\Delta \cos \theta_{\gamma \gamma}=0.8$ were again roughly estimated from the Serpukhov Primakoff experiment [7]; $0.1<\omega_{1}<0.6 \mathrm{GeV}$ and $-1<\cos \theta_{\gamma \gamma}<-0.2$, assuming similar acceptance at J-PARC. This gives the Primakoff rate of about $0.03 \times \varepsilon \mathrm{Hz}$, i.e. about $2.6 \mathrm{k} \times \varepsilon$ per day. Assuming $\varepsilon=0.5$, about 10k Primakoff events can be accumulated per week. Primakoff experiments usually be carried out with several different atomic number targets for systematic checks of target atomic number dependence of Primakoff event extraction. Expected Primakoff rate of 10k per week per target is thus feasible to complete data taking with several targets in a couple of months running time. In case of difficulties in $R \& D$ of the septum magnet or additional radiation shields, the pion beam energy can be compromised to $30 \sim 35 \mathrm{GeV}$ where abundant yields can be provided and the experiment is still feasible.

\section{References}

[1] J. Donoghue, E. Golowich, and B. Holstein, "Dynamics of the Standard Model” , Cambridge University Press (1992).

[2] U. Bürgi, Nucl. Phys B479, 392 (1997).

[3] J. Gasser, M.A. Ivanov, M.E. Sainio, Nucl. Phys. B745, 84 (2006).

[4] J. Roche et al., Phys. Rev. Lett. 85, 708 (2000), M. Schumacher, Prog. Part. Nucl. Phys. 55, 567 (2005), P.Bourgeois et al., Phys. Rev. Lett. 97, 212001 (2006).

[5] L.V. Fil'kov and V.L. Kashevarov, Phys. Rev. C73, 035210 (2006). 
[6] C. Berger et al. (PLUTO Collaboration), Z. Phys. C 26, 199 (1984), A. Courau et al. (DM1 Collaboration), Nucl. Phys. B271, 1 (1986), Z. Ajaltoni et al. (DM2 Collaboration), Phys. Lett. B194, 573 (1987), J. Boyer et al. (Mark II Collaboration), Phys. Rev. D 42, 1350 (1990).

[7] Yu. M. Antipov et al., Phys. Lett. B 121, 445 (1983); Z. Phys. C24, 39 (1984), Z. Phys. C 26, 495 (1985); Phys Rev. D 36, 21 (1987).

[8] J. Ahrens et al., Eur. Phys. J. A23, 113 (2005).

[9] J. Friedrich, these proceedings.

[10] http://j-parc.jp

[11] S. Sawada, et al., J-PARC Letter of Intent L14: Construction of a High Momentum Beam Line at the 50-GeV Proton Synchrotron (2002).

[12] J. L. Sandford and C. L. Wang, BNL report No. 11299 \& 11479 (1967).

[13] C. L. Wang, Phys. Rev. Lett. 25, 1068 (1970).

[14] A. Yamamoto, KEK Technical Report 81-13, (1983). 